\title{
Tyrosine Kinase Inhibitors (TKIs) for the Treatment of Concurrent Colorectal and Renal Cell Carcinomas
}

ISSN: 2637-7632

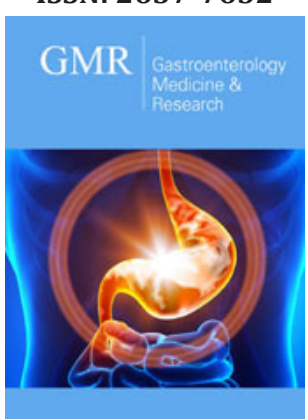

*Corresponding author: Athanasios Karampeazis, Department of Medical Oncology, 401 General Military Hospital of Athens, Greece

Submission: 制 September 23, 2020

Published: 皆 October 09, 2020

Volume 5 - Issue 2

How to cite this article: Vassiliki Kardara, Oraianthi Fiste, Athanasios Karampeazis. Tyrosine Kinase Inhibitors (TKIs) for the Treatment of Concurrent Colorectal and Renal Cell Carcinomas. Gastro Med Res. 5(2). GMR.000608. 2020.

DOI: $10.31031 /$ GMR.2020.05.000608

Copyright@ Athanasios Karampeazis, This article is distributed under the terms of the Creative Commons Attribution 4.0 International License, which permits unrestricted use and redistribution provided that the original author and source are credited.

\section{Vassiliki Kardara, Oraianthi Fiste and Athanasios Karampeazis*}

Department of Medical Oncology, 401 General Military Hospital of Athens, Greece

\section{Abstract}

Apart from representing relatively rare incidents, multiple primary malignancies pose, also, a therapeutic challenge. Treatment with anti-angiogenetic tyrosine kinase inhibitors have been proven to be effective in a variety of malignancies, including advanced renal cell and colorectal cancers. Herein, we report an interesting case of a 69-years-old male with renal cell carcinoma with pulmonary metastases and a synchronous localized colon cancer, who achieved significant responses to both cancers after firstline treatment with pazopanib and second-line treatment with axitinib. In the era of targeted therapies, angiogenesis inhibition may serve as an efficacious and well-tolerated therapeutic option in patients with coexisting colorectal and kidney tumors.

Keywords: Colorectal cancer; Renal cell carcinoma; Multiple primary malignancies; Tyrosine kinase inhibitors

\section{Introduction}

Renal cell carcinoma (RCC) represents the vast majority of all primary renal malignancies, with an all-age incidence around 4.4 cases per 100,000 persons, for both sexes [1]. The simultaneous occurrence of RCC and colorectal carcinoma (CRC) is quite uncommon, with prevalence rates from 0.03 to $4.8 \%$ [2]. We present the rare case of an elderly male with metastatic RCC and a synchronous adenocarcinoma of the hepatic flexure of the colon, who responded to treatment with anti-angiogenic receptor tyrosine kinase inhibitors (TKIs).

\section{Case Presentation}

A 69 years-old male, smoker, with a past history of hypertension, depression, benign prostatic hyperplasia, and hypercholesterolemia, presented with macroscopic hematuria and weight loss. Computed tomography scans revealed a left renal mass, bilateral pulmonary nodules, and thickening of the hepatic flexure colon mass. Patient underwent a left nephrectomy and the histopathological examination confirmed the presence of a grade 3, pT3Nx, clear-cell carcinoma. Colonoscopy identified a mass in the transverse colon covering three quarters of the lumen and the histological report confirmed the presence of a moderately differentiated invasive colon adenocarcinoma (Figure 1). Post surgery CT scans revealed deterioration of the known pulmonary lesions and presence of a new left pleural effusion (Figure 2a) together with a hepatic flexure colon mass (Figure 2b). A CT-guided lung lesion biopsy was consistent with RCC metastatic disease. In view of the asymptomatic colon cancer and the rapidly imaging deterioration of the metastatic renal cancer, treatment with pazopanib commenced with acceptable toxicity and partial response of both the renal metastatic disease and the colonic primary tumor. After eight months of treatment, imaging tests revealed disease progression in the lung, while the colon mass was further responding. Second-line treatment with axitinib commenced and the restaging CT scans, after 10 months from initial diagnosis, were consistent with a new partial response of metastatic disease (Figure 3a \& 3b), while a new colonoscopy revealed complete remission of the colon cancer. Treatment with axitinib continued for 5 months overall, with good tolerance. The patient presented brain and leptomeningeal metastatic disease with rapid clinical deterioration and succumbed 15 months after initial diagnosis. 


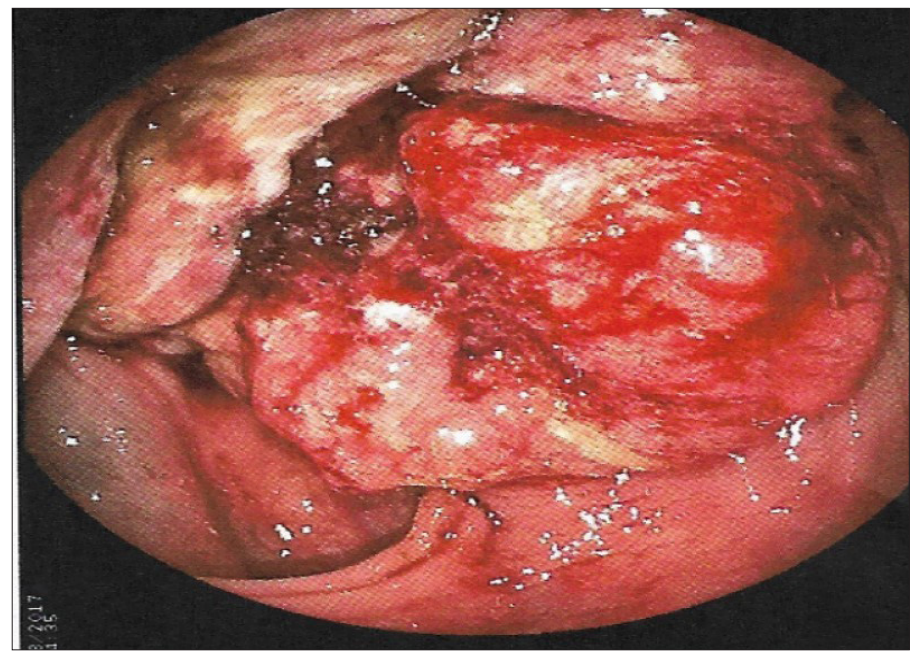

Figure 1: Colonoscopy photo of transverse colon tumour.

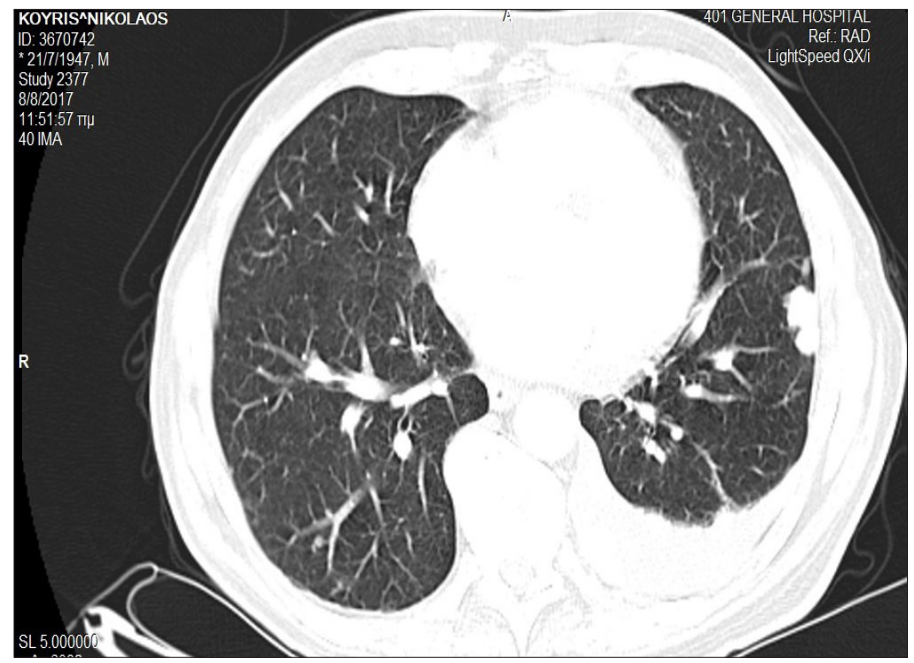

Figure 2a: A baseline chest CT scan showing multiple bilateral lung lesions and a left pleural effusion.

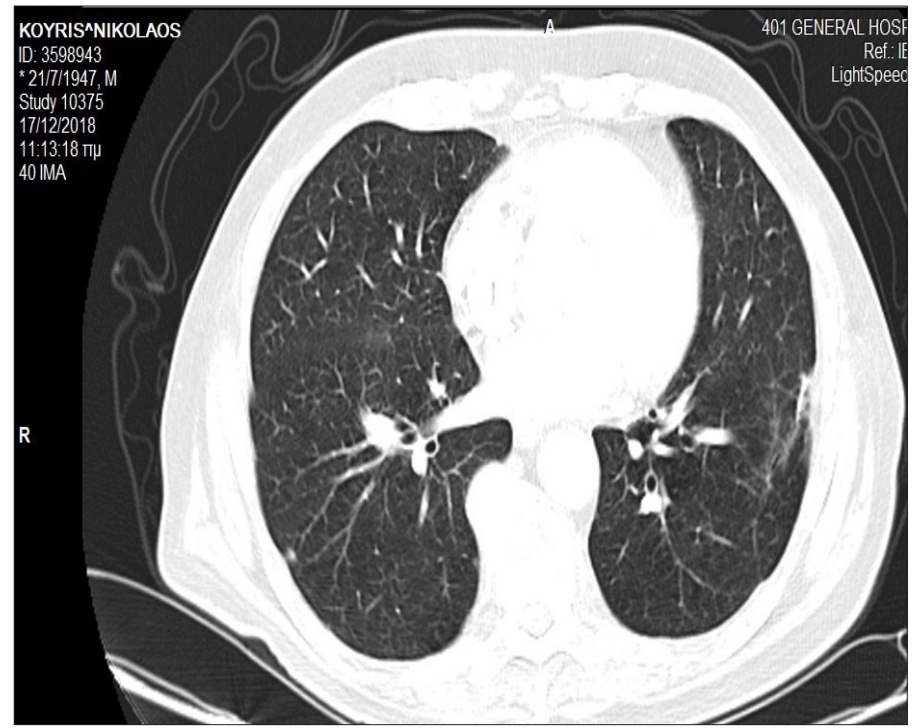

Figure 2b: A chest CT scan after 10 months of treatment with TKIs showing partial remission of lung metastatic disease. 


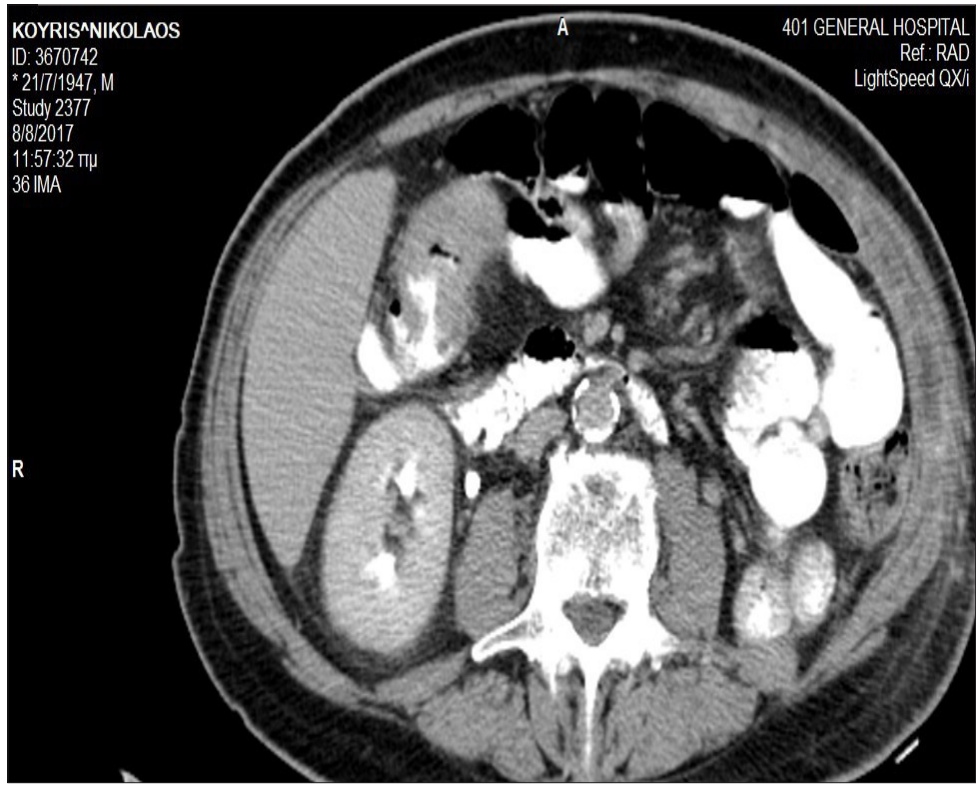

Figure 3a: A baseline abdomen CT scan showing thickening of the transverse colon.

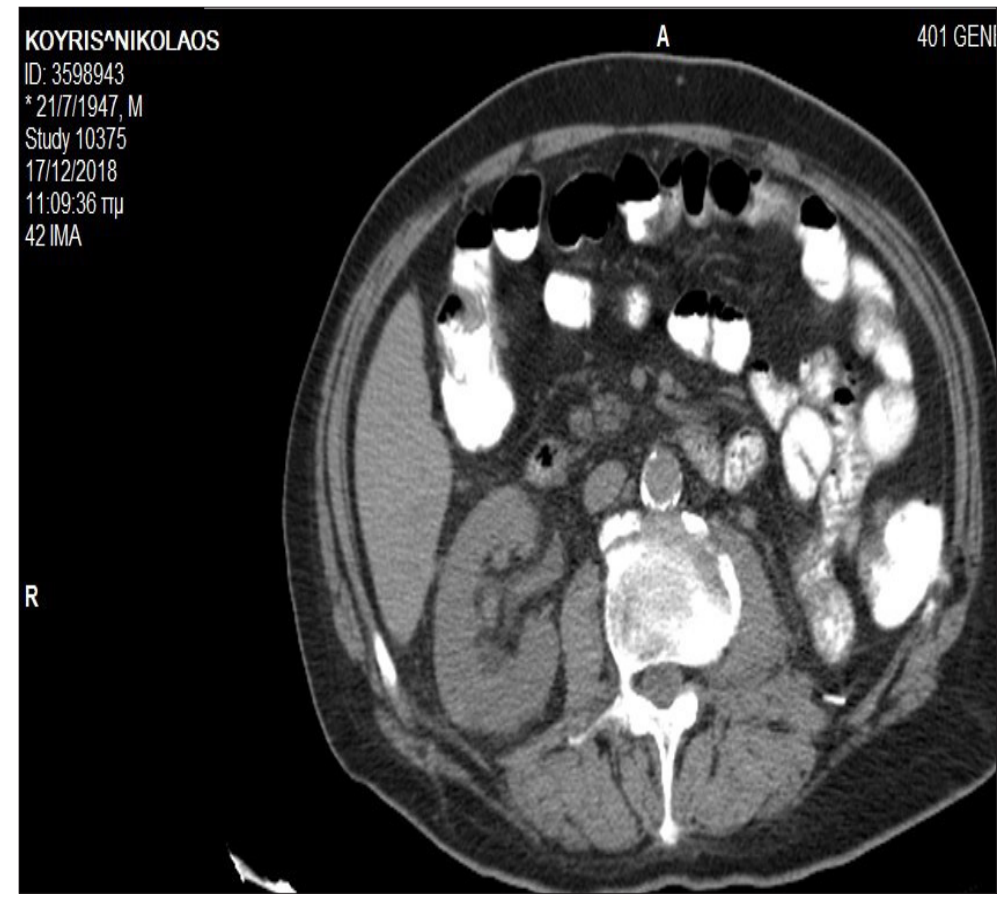

Figure 3b: An abdomen CT scan after 10 months of treatment with TKIs showing normal appearance of the transverse colon.

\section{Discussion}

Multiple primary malignancies (MPMs) arising in different sites, with different histological characteristics, in the same individual are classified into (a) synchronous malignancies when they occur within a 6-month period, and (b) metachronous when they have been developed after the 6-month interval [3]. Despite being relatively rare, with an approximate frequency of 2.4-
$8 \%$ in cancer populations, synchronous malignancies remain a therapeutic dilemma, since it is quite complicated to select the proper anticancer regimens for both cancers, in order to achieve optimal treatment benefit without increased toxicity. In addition, data regarding their therapeutic approaches depend mostly on published case reports and case series, given not only the exclusion of patients with MPMs from clinical trials, but also the absence of standard treatment guidelines [4]. 
In recent years, targeting angiogenesis has been the cornerstone of RCC treatment. Indeed, numerous TKIs have received United States Food and Drug Administration (FDA) approval in patients with advanced RCC, including sunitinib, sorafenib, pazopanib, axitinib, lenvatinib, and cabonzatinib. These are oral pharmaceutical drugs which inhibit the phosphorylation of the enzymes called receptor tyrosine kinases (RTKs), like the vascular endothelial growth factor receptor (VEGFR), the platelet-derived growth factor receptor (PDGFR), the fibroblast growth factor receptor (FGFR), and c-Kit, thus inducing anti-angiogenic and anti-proliferative effects [5]. In particular, pazopanib is a selective, second-generation, multikinase inhibitor of VEGFR-1, 2, 3, PDGFR- $\alpha, \beta$, and c-Kit [6], whilst axitinib is a highly selective, second-generation TKI with specificity for VEGFRs 1-3 [7]. Blocking angiogenesis, through several mechanisms, has also been demonstrated effective in CRC patients; bevacizumab is an anti- vascular endothelial growth factor (VEGF) antibody, aflibercept is a soluble decoy receptor that binds VEGF-A, VEGF-B, and placental growth factor (PIGF), and ramucirumab is an anti-VEGFR antibody [8]. Currently, regorafenib is the sole FDA approved anti-angiogenic TKI for refractory metastatic CRC, whereas the activity of pazopanib and axitinib in patients with advanced CRC has been reported in various phase I-II clinical studies [9-17], thus warranting further evaluation. Our patient was diagnosed with concurrent metastatic RCC and localized CRC, and upon informed consent he received pazopanib as first-line treatment and axitinib upon disease progression, resulting in longlasting response for both diseases. As far as we are aware, this is the first case report highlighting the activity of these TKIs in those malignancies occurring concurrently.

\section{Conflicts of Interest}

The authors have no conflict of interest and no financial disclosure.

\section{References}

1. Howlader N, Noone AM, Krapcho M, Garshell J, Miller D, et al. (2014) SEER cancer statistics review, 1975-2011, national cancer institute, based on November 2013 SEER data submission, Bethesda.

2. Capra F, Scintu F, Zorcolo L, Marongiu L, Casula G (2003) Synchronous colorectal and renal carcinomas. Is it a definite clinical entity? Chirurgia Italiana 55(6): 903-906.

3. Amer MH (2014) Multiple neoplasms, single primaries, and patient survival. Cancer Manag Res 6: 119-134.

4. Vogt A, Schmid S, Heinimann K, Frick H, Herrmann C, et al. (2017) Multiple primary tumours: Challenges and approaches, a review. ESMO Open 2(2): e000172.
5. Qin S, Li A, Yi M, Yu S, Zhang M, et al. (2019) Recent advances on antiangiogenesis receptor tyrosine kinase inhibitors in cancer therapy. J Hematol Oncol 12(1): 27.

6. Frampton JE (2017) Pazopanib: A review in advanced renal cell carcinoma. Target Oncol 12(4): 543-554.

7. Hu-Lowe DD, Zou HY, Grazzini ML, Hallin ME, Wickman GR, et al. (2008) Nonclinical antiangiogenesis and antitumor activities of axitinib (AG-013736), an oral, potent, and selective inhibitor of vascular endothelial growth factor receptor tyrosine kinases 1, 2, 3. Clin Cancer Res 14(22): 7272-7283.

8. Kircher SM, Nimeiri HS, Benson AB $3^{\text {rd }}$ (2016) Targeting angiogenesis in colorectal cancer: Tyrosine kinase inhibitors. Cancer J 22(3): 182-189.

9. Bennouna J, Deslandres M, Senellart H, de Labareyre C, Ruiz-Soto R, et al. (2015) A phase I open-label study of the safety, tolerability, and pharmacokinetics of pazopanib in combination with irinotecan and cetuximab for relapsed or refractory metastatic colorectal cancer. Invest New Drugs 33(1): 138-147.

10. Brady J, Corrie P, Chau I, Digumarti R, Adams LM, et al. (2013) An openlabel study of the safety and tolerability of pazopanib in combination with FOLFOX6 or CapeOx in patients with colorectal cancer. Invest New Drugs 31(5): 1228-1235.

11. Fu S, Hou MM, Naing A, Janku F, Hess K, et al. (2015) Phase I study of pazopanib and vorinostat: a therapeutic approach for inhibiting mutant p53-mediated angiogenesis and facilitating mutant p53 degradation. Ann Oncol 26(5): 1012-1018.

12. Plummer R, Madi A, Jeffels M, Richly H, Nokay B, et al. (2013) A Phase I study of pazopanib in combination with gemcitabine in patients with advanced solid tumors. Cancer Chemother Pharmacol 71(1): 93-101.

13. Grávalos C, Carrato A, Tobeña M, Rodriguez-Garrote M, Soler G, et al. (2018) A Randomized phase ii study of axitinib as maintenance therapy after first-line treatment for metastatic colorectal cancer. Clin Colorectal Cancer 17(2): e323-e329.

14. Infante JR, Reid TR, Cohn AL, Edenfield WJ, Cescon TP, et al. (2013) Axitinib and/or bevacizumab with modified FOLFOX-6 as first-line therapy for metastatic colorectal cancer: A randomized phase 2 study. Cancer 119(14): 2555-2563.

15. Bendell JC, Tournigand C, Swieboda-Sadlej A, Barone C, Wainberg ZA, et al. (2013) Axitinib or bevacizumab plus FOLFIRI or modified FOLFOX-6 after failure of first-line therapy for metastatic colorectal cancer: A randomized phase II study. Clin Colorectal Cancer 12(4): 239-247.

16. Sharma S, Abhyankar V, Burgess RE, Infante J, Trowbridge RC, et al. (2010) A phase I study of axitinib (AG-013736) in combination with bevacizumab plus chemotherapy or chemotherapy alone in patients with metastatic colorectal cancer and other solid tumors. Ann Oncol 21(2): 297-304.

17. Bendell JC, Joseph M, Barnes K, Mainwaring M, Shipley D, et al. (2017) A Phase-2 Trial of single agent axitinib as maintenance therapy following first-line treatment with modified FOLFOX/bevacizumab in patients with metastatic colorectal cancer. Cancer Invest 35(6): 386-392. 